\title{
Produção de couve brassica oleracea $L$. var. acephala com adubação mineral e orgânica
}

\section{Collard greens yield with mineral and organic fertilization}

\author{
Gilberto Yudi Shingo ${ }^{1}$; Maurício Ursi Ventura ${ }^{2 *}$
}

\section{Resumo}

Foi estudado o desenvolvimento da cultura da couve-de-folha na fase de muda e após o transplantio em vasos, submetida a diferentes tratamentos de adubação, os quais incluíam fertilizante mineral (como padrão), composto Bokashi (Bo) e EM-4, húmus de minhoca (Hu) e ácido pirolenhoso (AP), combinados em dez tratamentos para cada fase. O tratamento padrão incluiu substrato comercial para mudas (SC) e adubação após o transplantio com a fórmula 08-28-16. O comprimento do limbo foliar foi avaliado 25 dias após a emergência. O comprimento e largura do limbo foliar foram avaliados 85 dias após o transplantio. O peso fresco e seco do limbo e pecíolo foi avaliado aos 144 dias após a semeadura. Desenvolvimento inferior foi observado em plantas com Hu. De maneira geral, nos tratamentos com Bo e EM-4, os resultados foram similares ou apresentaram desenvolvimento próximo ao tratamento padrão.

Palavras-chave: Microorganismos eficazes, orgânico, ácido pirolenhoso

\begin{abstract}
The development of the collard greens crop was studied in the seedling phase and after planting in vessels, which received different treatments of fertilization which included mineral fertilizer (as standard), Bokashi compost (Bo) and EM4, earthworms humus (Hu), and piroligneous acid (AP), combined in ten treatments for each crop phase. The standard treatment included commercial substrate for seedlings (SC) and after planting with the formula 08-28-16. The length of the leaf was assessed 25 days after emergency. The leaf length and width was assessed 85 days after planting. The fresh and dry weight of leaf and petiole were assessed 144 days after seedling. Inferior development was observed in plants with humus. In general, in the treatments which included Bokashi, the results were similar or close to the standard treatment.
\end{abstract}

Key words: Effective microorganisms, organic, pyroligneous acid

\footnotetext{
1 Mestrando em Agronomia pela Universidade Estadual de Londrina; Fundação Mokiti Okada - MOA - Centro de Pesquisa. E-mail: gyshingo@yahoo.com.br.

2 Professor Doutor em Agronomia pela Universidade Estadual de Londrina; E-mail: mventura@uel.br.

* Autor para correspondência
} 


\section{Introdução}

A couve-de-folha (Brassica oleracea var. acephala) é, dentre as espécies cultivas, a que mais se assemelha à couve silvestre, não forma cabeça e suas folhas apresentam limbo bem desenvolvido, arredondado, com pecíolo longo e nervuras bem destacadas (FILGUEIRA, 2003). A produção comercial é normalmente conduzida segundo as recomendações convencionais, desde o preparo e correção do solo, adubações orgânica e química, até o controle de pragas e doenças (TAVARES; TRANI; SIQUEIRA, 1998). A colheita é iniciada aos 80-90 dias do transplante, perdurando por um período de oito meses. Filgueira (2003) refere-se ao padrão de 25-30 cm de comprimento para comercialização de folhas de couve.

$\mathrm{Na}$ linha de agricultura alternativa, o método da agricultura natural preconizado por Mokiti Okada em 1935, considera que a natureza é a mestra que devemos seguir, respeitando e agradecendo por tudo que ela nos proporciona. Entretanto, para viabilizar e acelerar o seu trabalho, Teruo Higa preconiza o uso de uma solução de microorganismos eficazes (EM4) e o composto farelado seco bokashi, no preparo do solo e na condução da cultura (FUNDAÇÃO MOKITI OKADA, 1999).

Em trabalho com couve-brócolos (B. oleracea L. var. italica) em sistema convencional, orgânico e natural em dois sistemas distintos (natural 1 com bokashi e natural 2 sem o uso do bokashi), as maiores produções foram obtidas nos tratamentos convencional e natural 1 (OTUTUMI; VENTURA; NEVES, 2001). Na réplica deste trabalho, o ataque à folha pela "vaquinha" Diabrotica speciosa foi significativamente reduzido nos sistemas alternativos (VENTURA; OTUTUMI; NEVES, 2006). Assim, estes sistemas atendem os anseios dos consumidores que buscam adquirir produtos livres de resíduos de inseticidas.

$\mathrm{Na}$ agricultura orgânica, solos com maior teor de matéria orgânica e atividade biológica geralmente apresentam boa fertilidade. A produção agrícola em tais solos apresenta baixa incidência de pragas, cuja redução pode ser atribuída ao baixo conteúdo de nitrogênio $(\mathrm{N})$ em culturas orgânicas. Práticas agrícolas, como o uso excessivo de fertilizantes inorgânicos, podem causar desequilíbrio nutricional (ALTIERI; NICHOLLS, 2003). As diferentes escolas de agricultura orgânica preconizam diferentes estratégias de fertilização dos solos. Várias alternativas são propostas de forma a substituir os adubos solúveis. A avaliação de diferentes fontes de adubação para os sistemas orgânicos é fundamental para o incremento da sua eficiência e competitividade.

O objetivo deste trabalho foi comparar o desenvolvimento da cultura da couve na fase de muda e após o transplantio em vasos, submetida aos diferentes tratamentos de adubação os quais incluíam fertilizante mineral (como padrão), composto Bokashi e EM-4, húmus de minhoca e ácido pirolenhoso, combinados em dez tratamentos para cada fase.

\section{Material e métodos}

O experimento foi instalado em casa-devegetação do Departamento de Agronomia da Universidade Estadual de Londrina. Utilizou-se a cultivar Geórgia da empresa Topseed. As mudas foram produzidas em 10 bandejas de isopor de 128 células cada e volume de $40 \mathrm{ml}$ de substrato por célula.

Numa primeira etapa estudou-se a produção de mudas com os tratamentos: T1 substrato comercial (SC); T2 99\% SC e 1\% de bokashi (Bo) Gardenbokashi (Korin Agropecuária Ltda., Ipeúna - SP); T3 99\% SC e 1\% Bo, tratado com EM4 (Korin Agropecuária Ltda., Ipeúna - SP); T4 30\% de casca de arroz carbonizada (Car) e 70\% de húmus de minhoca $(\mathrm{Hu})$; T5 30\% Car e 70\% Hu, tratado com ácido pirolenhoso (AP) (Carvoaria Coroados, Londrina, PR); T6 30\% Car, 69\% Hu e 1\% Bo; T7 $10 \%$ Car, $30 \%$ Hu e $60 \%$ de solo de cultivo (SoC); T8 10\% Car, $30 \%$ Hu e $60 \%$ de solo de mata (SoM), 
T9 97\% SoC e 3\% Bo, tratado com EM4; e T10 97\% SoM e 3\% Bo, tratado com EM4.

O bokashi é uma mistura de farelos vegetais fermentada com o EM4, com a função de favorecer a decomposição fermentativa de material (FUNDAÇÃO MOKITI OKADA, 1999). O EM-4 é uma composição líquida de lactobacilos, leveduras, actinomicetos, bactérias fotossintéticas e fungos filamentosos, com a função de produzir substâncias úteis à planta, tais como hormônios e vitaminas, através da captação da energia solar e ação sobre a matéria vegetal fresca e semi decomposta (FUNDAÇÃO MOKITI OKADA, 1999). O ácido pirolenhoso é um subproduto da carbonização da madeira obtido através da condensação da fumaça, decantação e destilação do extrato. Segundo Zanetti et al. (2004), o seu emprego pode potencializar a absorção de nutrientes em pulverizações foliares.

As mudas foram regadas diariamente. A variável avaliada nas mudas foi o comprimento do limbo foliar aos 25 dias da semeadura.
O transplante foi realizado 28 dias após a semeadura, quando as mudas apresentavam três folhas definitivas em vasos de barro com 5 litros de volume com $20 \mathrm{~cm}$ de diâmetro e um orifício central para drenagem no seu fundo. As adubações foram feitas com o propósito de dar continuidade aos sistemas utilizados na formação das mudas, sendo a química conforme recomendado por Filgueira (2003) e a natural conforme Fundação Mokiti Okada (1999), apresentadas na Tabela 1. Utilizou-se solo de barranco, devido à sua baixa fertilidade natural, para que os resultados finais pudessem evidenciar a diferença entre tratamentos. Antes da instalação do experimento, o solo apresentava a seguinte caracterização química: $\mathrm{pH} \mathrm{CaCl2:} \mathrm{4,5;} \mathrm{Carbono:}$ $1,527 \mathrm{~g} / \mathrm{kg}$; M.O.: 2,626 g/kg; Al: 0,51 Cmolc/dm³; Mg: 2,3 Cmolc/dm ${ }^{3}$ Ca: $2,7 \mathrm{Cmolc} / \mathrm{dm}^{3} ; \mathrm{H}+\mathrm{Al}$ : 4,96 Cmolc/dm³ K: 0,015 Cmol/ $\mathrm{dm}^{3}$; P: $3,3 \mathrm{mg} /$ $\mathrm{dm}^{3}$; CTC: $9,975 \mathrm{Cmolc} / \mathrm{dm}^{3}$; e V\%: 50,27.

Tabela 1. Tratamentos utilizados para adubação de couve cultivada em vasos com solo de barranco, em casa-devegetação. Londrina, junho de 2007.

\begin{tabular}{lcc}
\hline \multicolumn{1}{c}{ Tratamentos no vaso } & \multicolumn{2}{c}{ Dosagens } \\
\cline { 2 - 3 } & g/vaso*** & diluição \\
\hline SC + 08-28-16 & 40 & - \\
SCBo + Bo & 25 & - \\
SCBoEM4 + Bo EM4* & 25 & $0,2 \%$ \\
CarHu + Hu & 120 & - \\
CarHuAP + Hu AP* & 120 & $0,2 \%$ \\
CarHuBo + Hu Bo** & $120+25$ & - \\
CarHuSoC + Hu & 120 & - \\
CarHuSoM + Hu & 120 & - \\
SoCBoEM4 + Bo EM4* & 25 & $0,2 \%$ \\
SoMBoEM4 + Bo EM4* & 25 & $0,2 \%$ \\
\hline
\end{tabular}

$\mathrm{SC}=$ substrato convencional; $\mathrm{Car}=$ casca de arroz carbonizada; $\mathrm{Bo}=$ bokashi; $\mathrm{Hu}=$ húmus de minhoca; $\mathrm{AP}=$ ácido pirolenhoso; EM4= solução de microorganismos benéficos; $\mathrm{SoC}=$ solo de cultivo; $\mathrm{SoM}=$ solo de mata.

* Insumos aplicados em diluição.

** Aplicação conjunta de húmus e bokashi.

*** Dosagens de adubo formulado ou húmus ou bokashi. 
Nos vasos, o ácido pirolenhoso foi aplicado semanalmente $(0,2 \%)$ através de regas e o EM4 $(0,2 \%)$ através de pulverização. Os demais tratamentos receberam regas e pulverizações com o mesmo volume de solução, sem os insumos. O tratamento com o adubo formulado 08-28-16 recebeu uma aplicação de $2 \mathrm{~g}$ de uréia por vaso, superficial no solo, aos 85 dias após a semeadura. O bokashi foi aplicado nos tratamentos pré-determinados, na dosagem de 1,5 g superficialmente via solo, 88 dias após a semeadura.

Visando o controle de pulgões, aplicouse semanalmente o óleo de nim a 0,3\% após o transplantio.

Aos 85 dias após o plantio, realizou-se a medição do comprimento e largura do limbo foliar, selecionando-se a $4^{\mathrm{a}}$. folha mais nova. Aos 122 dias da semeadura foram coletadas quatro folhas por parcela, descartando-se as folhas baixeiras e injuriadas. Considerou-se para a coleta o tamanho comercial (25-30 cm de comprimento de limbo foliar). No tratamento CarHuSoc $+\mathrm{Hu}$ não foi feita colheita porque duas plantas secaram e as outras não se desenvolveram o suficiente para se determinar o peso. As folhas foram acondicionadas em sacos de papel. No laboratório foram limpas e com um estilete o limbo foi separado do pecíolo. Ambas as partes foram pesadas em balança semianalítica, para determinação do peso fresco. Depois, foram lavadas em água corrente para retirar poeira e mergulhadas por 2 segundos em água destilada e acondicionadas em seguida sobre papel jornal por 1 hora para secagem. A seguir, foram novamente ensacadas e levadas para secagem em estufa por 72 horas a $55^{\circ} \mathrm{C}$. Após este período foi determinado o peso seco.
Os experimentos foram instalados em delineamento inteiramente casualizado. Realizouse análise de variância e teste de Tukey (5\%) para comparação das médias.

\section{Resultados e discussão}

Nas mudas, o comprimento do limbo foliar (CLb) foi maior nos tratamentos $\mathrm{SC}, \mathrm{SC}+\mathrm{Bo}, \mathrm{SC}$ + Bo + EM4, SoM + Bo (valores médios acima de $4 \mathrm{~cm}$ ), do que nos tratamentos $\mathrm{Car}+\mathrm{Hu}$ e $\mathrm{Car}+$ $\mathrm{Hu}+\mathrm{SoC}$, (aproximadamente 2,6 cm) (Tabela 2). Valores intermediários, próximos de $3 \mathrm{~cm}$, foram observados nos tratamentos CarHuAp, CarHuBo, CarHuSoM e SoCBoEM4.

Nos vasos, no tratamento SC + 08-28-16 foram verificados valores de CLv superiores do que nos tratamentos $\mathrm{CarHu}+\mathrm{Hu}$, CarHuAP + HuAP, CarHuSoC + Hu. Nos demais tratamentos foram verificados valores similares a $\mathrm{SC}+08$ 28-16. O tratamento SomBoEM4 + BoEM4 foi o que mais se aproximou de $\mathrm{SC}+08-28-1$ (CLv apenas $8,74 \%$ menor). No entanto, aos 85 dias da semeadura, nenhum dos tratamentos apresentou o comprimento do limbo no padrão comercial (25$30 \mathrm{~cm}$ ), o que pode ter ocorrido, devido à limitação no desenvolvimento radicular do cultivo em vaso, tendendo ao enovelamento das radicelas na parede interna. Para a largura do limbo (LLv), os tratamentos $\mathrm{CarHu}+\mathrm{Hu}$, CarHuAp $+\mathrm{HuAp}$, CarHuSoc + $\mathrm{Hu}$ e CarHuSoM $+\mathrm{Hu}$, foram significativamente inferiores a $\mathrm{SC}+08-28-16$.

No tratamento CarHuSoC $+\mathrm{Hu}$ não foi possível fazer a avaliação das variáveis PFL, PSL, PFP e PSP no vaso, após o transplantio, porque as plantas morreram ou apresentaram desenvolvimento muito reduzido (Tabela 3 ). 
Tabela 2. Comprimento do limbo foliar (CLb e CLv - bandeja e vaso) de couve aos 25 e 85 dias após a semeadura, respectivamente, e largura do limbo foliar (LLv) aos 85 dias após a semeadura. Londrina, 2007.

\begin{tabular}{ccccc}
\hline $\begin{array}{c}\text { Tratamento bandeja } \\
\text { (25 dias) }\end{array}$ & CLb (cm) & Tratamento vaso (85 dias) & CLv (cm) & LLv (cm) \\
\hline SC & $4,0700 \mathrm{a}$ & SC+08-28-16 & $20,220 \mathrm{a}$ & $16,100 \mathrm{a}$ \\
SCBo & $4,0325 \mathrm{ab}$ & SCBo + Bo & $16,940 \mathrm{ab}$ & $12,660 \mathrm{ab}$ \\
ScBoEM4 & $4,4675 \mathrm{a}$ & SCBoEM4 + BoEM4 & $16,720 \mathrm{abc}$ & $12,800 \mathrm{ab}$ \\
CarHu & $2,6175 \mathrm{c}$ & CarHu $+\mathrm{Hu}$ & $14,400 \mathrm{bc}$ & $9,920 \mathrm{bc}$ \\
CarHuAP & $2,9825 \mathrm{bc}$ & CarHuAP $+\mathrm{HuAP}$ & $14,960 \mathrm{bc}$ & $11,420 \mathrm{bc}$ \\
CarHuBo & $2,9675 \mathrm{bc}$ & CarHuBo $+\mathrm{HuBo}$ & $17,180 \mathrm{ab}$ & $13,040 \mathrm{ab}$ \\
CarHuSoC & $2,6400 \mathrm{c}$ & CarHuSoC $+\mathrm{Hu}$ & $11,780 \mathrm{c}$ & $8,380 \mathrm{c}$ \\
CarHuSoM & $2,9600 \mathrm{bc}$ & CarHuSoM $+\mathrm{Hu}$ & $15,520 \mathrm{abc}$ & $11,420 \mathrm{bc}$ \\
SoCBoEM4 & $3,2125 \mathrm{abc}$ & SoCBoEM4 + BoEM4 & $16,700 \mathrm{abc}$ & $12,460 \mathrm{ab}$ \\
SoMBoEM4 & $4,3850 \mathrm{a}$ & SoMBoEM4 + BoEM4 & $17,680 \mathrm{ab}$ & $13,140 \mathrm{ab}$ \\
\hline C.V. & 16,6338 & & 14,7184 & 14,8240 \\
\hline
\end{tabular}

Médias com a mesma letra na coluna não diferem significativamente pelo teste Tukey a 5\% de probabilidade.

$\mathrm{SC}=$ substrato comercial; $\mathrm{Car}=$ casca de arroz carbonizada; $\mathrm{Hu}=$ húmus; $\mathrm{AP}=$ ácido pirolenhoso; $\mathrm{Bo}=$ bokashi; $\mathrm{Soc}=\mathrm{solo}$ de cultivo; SoM= solo de mata; EM4= solução de microrganismos benéficos.

Tabela 3. Peso fresco e seco do limbo (PFL e PSL) e peso fresco e seco do pecíolo (PFP e PSP) de couve, 150 dias após a semeadura. Londrina, 2007.

\begin{tabular}{ccccc}
\hline Tratamentos & PFL $(\mathbf{g})$ & PSL $(\mathbf{g})$ & PFP $(\mathbf{g})$ & PSP $(\mathbf{g})$ \\
\hline SC+08-28-16 (+ uréia) & $28,430 \mathrm{a}$ & $4,1550 \mathrm{a}$ & $24,728 \mathrm{a}$ & $2,7200 \mathrm{a}$ \\
SCBo + Bo & $16,578 \mathrm{~b}$ & $3,1800 \mathrm{a}$ & $10,003 \mathrm{~b}$ & $1,6600 \mathrm{a}$ \\
SCBoEM4 + BoEM4 & $19,388 \mathrm{ab}$ & $4,4625 \mathrm{a}$ & $14,485 \mathrm{~b}$ & $2,4475 \mathrm{a}$ \\
CarHu + Hu & $11,708 \mathrm{~b}$ & $2,4025 \mathrm{a}$ & $7,293 \mathrm{~b}$ & $1,1800 \mathrm{a}$ \\
CarHuAP + HuAP & $13,773 \mathrm{~b}$ & $3,2350 \mathrm{a}$ & $8,413 \mathrm{~b}$ & $1,4200 \mathrm{a}$ \\
CarHuBo + HuBo & $20,685 \mathrm{ab}$ & $4,4750 \mathrm{a}$ & $13,308 \mathrm{~b}$ & $2,5200 \mathrm{a}$ \\
CarHuSoM + Hu & $14,465 \mathrm{~b}$ & $3,5525 \mathrm{a}$ & $7,808 \mathrm{~b}$ & $1,4925 \mathrm{a}$ \\
SoCBoEM4 + BoEM4 & $17,703 \mathrm{~b}$ & $4,0350 \mathrm{a}$ & $13,133 \mathrm{~b}$ & $2,2475 \mathrm{a}$ \\
SoMBoEM4 + BoEM4 & $16,875 \mathrm{~b}$ & $3,5150 \mathrm{a}$ & $10,250 \mathrm{~b}$ & $1,7850 \mathrm{a}$ \\
\hline C.V. & 23,558 & 32,199 & 30,170 & 33,395 \\
\hline
\end{tabular}

$\mathrm{SC}=$ substrato comercial; $\mathrm{Bo}=$ bokashi; $\mathrm{EM} 4=$ solução de microrganismos benéficos; $\mathrm{Car}=$ casca de arroz carbonizada; Hu= húmus; $\mathrm{AP}=$ ácido pirolenhoso; $\mathrm{SoM}=$ solo de mata; $\mathrm{SoC}=$ solo de cultivo

Médias com a mesma letra na coluna, não diferem significativamente pelo teste Tukey a 5\% de probabilidade.

No tratamento $\mathrm{SC}+$ 08-28-16 foram verificados valores superiores para PFL do que os demais tratamentos, excetuando-se SCBoEm4 + BoEM4 e CarHuBo + HuBo (Tabela 3). Para o PSL não foram verificadas diferenças estatísticas entre os tratamentos. O percentual de peso seco em relação ao fresco foi de $14 \%$ para o tratamento $\mathrm{SC}+08-28$ 16 (menor percentual) e $24,53 \%$ para o tratamento $\mathrm{CarHuSoC}+\mathrm{Hu}$ (maior percentual).
Em relação ao PFP, o valor obtido em $\mathrm{SC}+08-$ 28-16 foi superior a todos os demais tratamentos. Entretanto, para o PSP, não foram constatadas diferenças estatísticas. Também neste caso, o menor percentual de acúmulo de matéria seca foi no tratamento $\mathrm{SC}+08-28-16(11 \%)$ e também o maior foi observado no tratamento $\mathrm{CarHuSoC}+\mathrm{Hu}$ $(19,08 \%)$. 
As discrepâncias entre peso fresco e peso seco ocorreram devido ao teor de umidades das folhas, sendo que o tratamento $\mathrm{SC}+08-28-16$ foi o que acumulou menos matéria seca.

Assim, o tratamento com adubação mineral apresentou valores mais próximos de comprimento de limbo foliar exigidos pelo mercado, porém, quanto ao acúmulo de matéria seca não foi significativamente melhor.

$\mathrm{O}$ húmus de minhoca como fertilizante $(\mathrm{CarHu}$ $+\mathrm{Hu}$ e CarHuSoC $+\mathrm{Hu}$ ), nas quantidades utilizadas neste trabalho, não proporcionou condições satisfatórias de desenvolvimento às plantas. Entretanto, verificou-se incremento médio de 13,$7 ; 3,8 ; 15,0 ; 17,6 ; 34,0 ; 15,0$ e $20,0 \%$ para as variáveis CL (muda e vaso), LL, PFL, PSL, e PFP e PSP, respectivamente, quando se adicionou o AP ao húmus (tratamento $\mathrm{CarHu}$ $+\mathrm{Hu}$ x CarHuAP + HuAP) (Tabelas 2 e 3). No tratamento CarHuAp + HuAp as folhas obtidas apresentaram aparência condizente com o padrão de comercialização no que se refere à coloração, o que não foi observado no tratamento $\mathrm{CarHu}+\mathrm{Hu}$. No tratamento $\mathrm{CarHu}+\mathrm{Hu}$ observou-se machas roxas nas folhas, características de deficiência de fósforo. Os resultados no incremento da produção e qualidade do produto quando se adicionou o AP ao húmus, corroboram resultados prévios com alface e quiabo, culturas nas quais se observou incrementos de até $30 \%$ no produto comercializável quando se adicionou o AP ao composto orgânico (MASCARENHAS et al., 2006).

\section{Conclusões}

Com as fontes e quantidades de materiais alternativos de adubação utilizados no trabalho, verificou-se que nos tratamentos nos quais se utilizou o composto Bokashi e o EM-4 o desenvolvimento das plantas foi, de maneira geral, similar à adubação mineral.

\section{Agradecimentos}

Pela dedicação e cooperação no desenvolvimento dos trabalhos, agradeço aos professores do curso de Agronomia, aos técnicos Davi, João, Márcio e Geraldo, ao encarregado Bié e aos auxiliares Cícero e Irmão.

\section{Referências}

ALTIERI, M. A.; NICHOLLS, C. I. Soil fertility management and insect pests: harmonizing soil and plant health in agroecosystems. Soil and Tillage Research, Berkeley, v. 72, n. 2, p. 203-211, 2003.

FILGUEIRA, F. A. R. Novo manual de olericultura: agrotecnologia moderna na produção e comercialização de hortaliças. 2. ed. Viçosa: UFV, 2003. p. 274-294.

FUNDAÇÃO MOKITI OKADA - FMO. Microorganismos eficazes EM na agricultura. São Paulo: FMO, 1999. 30 p.

MASCARENHAS, M. H. T.; LARA, J. F. R.; PURCINO, H. M. A.; SIMÕES, J. C.; MOREIRA, D. C.; FACION, C. E. Efeito da utilização de ácido pirolenhoso na produtividade de alface. In: CONGRESSO BRASILEIRO DE OLERICULTURA, 46., 2006, Goiânia. Anais... Goiânia: UFG, 2006. p. 3122-3125.

OTUTUMI, A.; VENTURA, M. U.; NEVES, P. M. O. J. Parâmetros agronômicos em couve-brócolos (Brassica oleracea L. var. italica) em sistema convencional, orgânico e natural. Semina, Londrina, v. 22, n. 2, p. 161164, jul/dez. 2001.

TAVARES, M.; TRANI, P. E.; SIQUEIRA, W. J. Couve Brassica oleracea L. var. acephala. In: FAHL, J. I.; CAMARGO, M. B. P.; PIZZINATTO, M. A.; BETTI, J. A.; MELO, A. M. T.; DEMARIA, I. C.; FURLANI, A. M. C. Boletim IAC 200: instruções agrícolas para as principais culturas econômicas. 6. ed. Campinas: Instituto Agronômico, 1998. p. 201-202.

VENTURA, M. U.; OTUTUMI, A.; NEVES, P. M. O. Feeding preference of Diabrotica speciosa (Ger.) (Coleoptera: Chrysomelidae) by broccoli leaves from natural, organic and conventional farming systems. Semina, Londrina, v. 27, n. 1, p. 43-46, jan/mar. 2006.

ZANETTI, M.; CAZETTA, J. O.; MATTOS JÚNIOR, D.; CARVALHO, S. A. Influência do extrato pirolenhoso na calda de pulverização sobre o teor foliar de nutrientes em limoeiro cravo. Revista Brasileira de Fruticultura, Jaboticabal, v. 26, n. 3, p. 529-533, dez. 2004. 